FERMILAB-Pub-00/174-T

June 19, 2000

\title{
Topology in the Bulk: Gauge Field Solitons in Extra Dimensions
}

\author{
Christopher T. Hill ${ }^{1}$ \\ and \\ Pierre Ramond ${ }^{2}$ \\ ${ }^{1}$ Fermi National Accelerator Laboratory \\ P.O. Box 500, Batavia, Illinois 60510, USA* \\ ${ }^{2}$ Institute for Fundamental Theory, \\ Physics Department, University of Florida \\ Gainesville, Florida, 32611 ${ }^{\dagger}$
}

\begin{abstract}
Certain static soliton configurations of gauge fields in $4+1$ dimensions correspond to the instanton in 4 Euclidean dimensions "turned on its side," becoming a monopole in $4+1$. The periodic instanton solution can be used with the method of images to construct solutions satisfying the brane boundary conditions. The $\theta$-term on the brane becomes a topological current source, yielding an emission amplitude for monopoles into the bulk. Instantons have a novel reinterpretation in terms of monopole exchange between branes.
\end{abstract}

*e-mail: hill@fnal.gov, ramond@phys.ufl.edu;

${ }^{\dagger}$ supported in part by the Department of Energy under grant DE-FG02-97ER41029 


\section{Introduction}

The possibility that extra space-time dimensions may be associated with lower energy scales, $\sim 1 \mathrm{TeV}$, rather than $M_{\text {Planck }}$ has become an active area of discussion in the past few years. Though many nagging theoretical questions abound in these schemes, e.g., "how do they match onto a string theory at higher energies ?," they do potentially offer some intriguing new possibilities for extensions beyond the Standard Model, and would have enormous significance for experiment at very high, albeit accessible, energies.

Models of extra-dimensions at the $\sim \mathrm{TeV}$ scale fall into two categories, those with gravity only in the bulk [1,2], and those that include gauge fields, as well as fermions, in the bulk [3]. It has been argued that the latter models can unify, in principle, at much lower scales than the usual GUT scale, due to the dimensional coupling constant and the accelerated power law running. In fact, this unification occurs generally for strong coupling and we expect strong dynamics in these schemes at the string scale [4]. Many phenomenological constraints have been placed upon the scales of the new extra dimension(s), and a compactification scale lower limit of order $\sim 1 \mathrm{TeV}$ seems to be indicated.

In the present paper we wish to discuss another feature of gauge fields in the bulk which is potentially important to the phenomenology, and perhaps the viability of these schemes. Extra dimensions with bulk gauge fields necessarily imply the existence of new physical, topologically stable static soliton solutions. The solitons we consider presently are composed only of the gauge degrees of freedom that are extended to the bulk, and do not involve any Higgs fields at high energies. We will confine our attention at present to $4+1$ bulk space-time dimensions.

The salient new feature in extending beyond $3+1$ dimensions to, e.g., $4+1$, is that the homotopy class $\Pi_{3}(S U(2))$, previously associated with the instanton winding number, now becomes associated with "magnetic charge." While instantons are quasi-localized "events" of finite action in $3+1[5,6]$, the monopoles are static particles, "world-lines" in $4+1$, and have a mass determined by the compactification scale. (In $4+1$ dimensions the gauge coupling constant

has mass dimension $-1 / 2$, and can be written as $g=g_{0} / \sqrt{M_{c}}$, where $g_{0}$ is 
dimensionless; $M_{c}$ is the compactification scale.)

All nonabelian Yang-Mills gauge groups, including those of the Standard Model, admit $S U(2)$ subgroups. All $S U(2)$ gauge theories have instanton solutions, when these theories are considered in Euclidean $d=4$. These are "pseudoparticle solutions" of the pure Euclidean gauge equations of motion, representing the homotopy group $\Pi_{3}(S U(2))$ [5], and correspond physically to WKB tunneling solutions in the quantum theory [6]. The tunneling is associated with the violation of a fermionic chiral charge, connected to the index of the instanton through the axial anomaly. In the case of QCD this charge is the $U(1)_{A}$ axial charge, and the instantons produce large tunneling amplitudes near the QCD scale, leading to an explicit breaking of the chiral $U(1)_{A}$ symmetry and the removal of an unwanted low mass Nambu-Goldstone boson from the spectrum [6]. However, the overall $U(1)_{A}$ phase of the quark mass-matrix is now elevated to a problem for QCD, the $\theta$ problem, and to date the resolution of this problem is unknown. In the case of the electroweak $S U(2)_{L}$, the charge is $B+L$, and the instantons, which have very small amplitudes, lead to an unobservable low rate of proton decay. Thus instantons, at least in QCD, play an important role in the physics of the Standard Model.

If Standard Model gauge fields, either those of QCD or $S U(2)_{L}$, can live in the bulk, we see immediately that there is a new aspect of the usual instanton. The instanton, which was previously a pseudoparticle on a Euclideanized $3+1$ brane, becomes a static, real massive particle, in the bulk. It becomes a magnetic monopole (though the magnetic charge will not necessarily be electromagnetic; in the QCD case it is chromomagnetic). The two-form field strength is dual to a 3 -form in $4+1$. However, if we contract the three-form dual with the time-like vector defining the solution's rest-frame, then there is a self-duality, the remnant of self-duality of the $d=4$ instanton. The topological charge is the space integral of a 4-form time component. Self-duality implies that the mass of the monopole is therefore determined; this object is a generalized magnetic monopole, and shares many features in common with the BPS monopole in $3+1$.

This new object has a modulus, the parameter associated with the size of the remnant instanton. If the theory is scale invariant, then this quantity is undetermined, and we have a one-parameter family of degenerate solutions 
of monopoles varying sizes. In the scale-invariant limit the monopoles have a common mass, $8 \pi^{2} M_{c} / g_{0}^{2}$ where $M_{c}$ is the compactification scale, and $g_{0}$ a dimensionless gauge coupling.

An immediate complication arises when we wish to consider monopoles in the bulk bounded by branes. Gauge fields must satisfy certain boundary conditions on the branes, and we must obtain self-dual monopole solutions consistent with these. Fortunately, there exist exact self-dual solutions for multi-instantons on a line [7], and these can be used with the method of images to construct selfdual monopoles consistent with the boundary conditions of the branes. Again, self-duality is powerful, and the interaction energy of the monopoles with the branes, or with each other, is zero.

As an application, we consider the $\mathrm{QCD} U(1)_{A}$ problem as viewed from a $4+1$ theory. The $\theta$-term is not Lorentz invariant in $4+1$ dimensions, and becomes a topological current source from the brane into the bulk, the analogue of a thermal "cathode current" in a vacuum tube. Instantons still exist in the theory, appearing now as cylindrical solutions, analogous to vortices, independent of $x^{5}$, in the Euclidean 5-dimensions that interconnect branes. In QCD, chiral charge can be removed from brane I by the instanton "vortex" and transported to brane II. The instantons, however, can be reinterpreted as emission/absorption vertices for monopoles into the bulk (with zero extant in $x^{5}$ ), and the instanton vortex as the "t-channel" exchange of a monopole between branes. The emission amplitude is now $\sim \mathcal{O}(1)$ rather than $\sim \exp \left(-8 \pi^{2} / g_{0}^{2}\right)$. The 't Hooft nonperturbative factor $\exp \left(-8 \pi^{2} / g_{0}^{2}\right)$ is restored by the exchange of monopoles "shining" from one brane to another. This produces a Yukawa factor $\exp (-M \delta)=\exp \left(-8 \pi^{2} / g_{0}^{2}\right)$, where $\delta$ is the interbrane spacing.

We construct an effective monopole Lagrangian in the bulk. Monopoles couple to the branes via instantons, allowing the exchange of topological charge between brane and monopoles in the bulk. We show how a nontrivial monopole condensate forms between the branes, which can support the topological current. The $\theta$-potential is obtained of the form $\propto \Lambda^{4} \exp (-M \delta) \cos \theta$, which represents the monopole condensate ("space charge") response to the applied current.

This picture bears some resemblance to the AdS-CFT correspondence in 
string theory. The boundary theory of point-like, or 0-dimensional instantons, is equivalent at low energies to a bulk theory of monopoles (1-dimensional worldlines). It also suggests a dimensional sequence of physical objects that are correlated with anomalies, i.e., instanton in $d$ dimensions and $\theta$-term becomes a monopole in $d+1$ with $\theta$-current.

\section{$24+1$ Solitons based Upon Instantons}

The arena of this investigation is a $d=4+1$ spacetime (the bulk) in which two parallel branes, defining $d=3+1$ internal worlds, are immersed. The ordinary spacetime coordinates are labeled by $x^{\mu}, \mu=0,1,2,3$, and the fifth dimension by $x^{5}$ (to avoid confusion with $x^{4}=i c t$ ). The branes are respectively located at I: $x^{5}=\delta_{I}$ and II: $x^{5}=\delta_{I I}$, with a constant interbrane separation $\delta=\delta_{I I}-\delta_{I}$.

We first consider a pure $S U(2)$ gauge theory which lives in the bulk, momentarily ignoring fermions. It is defined by the covariant derivative $D_{A}=$ $\partial_{A}+i A_{A}^{a} \tau^{a} / 2$, with field strengths $i \tau^{a} / 2 F_{A B}^{a}=\left[D_{A}, D_{B}\right]$, where capital letters denote the bulk coordinates, $A, B=0,1,2,3,5$, and $a, b=1,2,3$ are the $S U(2)$ labels. The canonical mass dimension of the vector potential in $4+1$ dimensions is $3 / 2$, and the coupling constant must have dimension $-1 / 2$. We introduce a scale $M_{c}$ and define the scale invariant coupling constant $g \sqrt{M_{c}} \equiv g_{0} . M_{c}$ becomes the compactification scale, and $g_{0}$ the dimensionless coupling constant in the effective $3+1$ theory at low energies. Note that the dual field strength in $4+1$ dimensions is the three-form:

$$
\widetilde{F}_{A B C}^{a}=\frac{1}{2} \epsilon_{A B C D E} F^{a D E}
$$

Define $\chi_{A}$ to be a spacelike 5-vector normal to the branes. Then a necessary gauge-invariant prescription of the gauge field boundary conditions on a brane is

$$
\chi_{A} F^{a A B}=0, \quad \text { at } x^{5}=\delta_{I, I I} .
$$

This removes unwanted gauge invariant vector field strengths in the $3+1$ theory.

The effective $3+1$ brane dual becomes $\widetilde{F}^{a A B}=\chi_{C} \widetilde{F}^{a A B C}$. The simplest gauge 
choice realizing these boundary conditions is to impose Neumann conditions for $A_{\mu}^{a}$ with $\mu=0,1,2,3$, i.e. $\partial A_{\mu}^{a} / \partial x^{5}=0$, at $x_{5}=\delta_{I, I I}$, and Dirichlet conditions for the $3+1$ "scalars" $A_{5}^{a}$, i.e. $A_{5}^{a}=0$ at $x_{5}=\delta_{I, I I}$. The lowest energy physical $A_{i}$ modes are zero-modes, independent of $x_{5}$, and form the usual $3+1$ gauge field. The effective theory, truncating of these modes and integrating over $x_{5}$ yields the normal $3+1$ Lagrangian with coupling constant $g_{0}$ and where $M_{c} \delta=1$. The solutions with nontrivial $x_{5}$ dependence, including $A_{5}$, are Kaluzsa-Klein modes; hence the boundary conditions neatly lift $A_{5}^{a}$ to a large mass, which would otherwise remain as an unwanted scalar excitation in the low energy $3+1$ theory. It follows that at short distances in the bulk we have approximately a continuum, scale invariant, $4+1$ dimensional gauge theory.

With nonabelian gauge fields in the bulk and two parallel branes there arises the possibility of topologically nontrivial Wilson line connections between the branes [8]. The branes are analogous to a Josephson junction. The Wilson line phase for an $S U(2)$ theory can be topologically nontrivial throughout the space-time of the bulk:

$$
\Omega_{I, I I}=P \exp \left(i g \int_{I}^{I I} d x^{5} A_{5}^{a} \frac{\tau^{a}}{2}\right)
$$

For example, for large $r$ in 3 space dimensions on brane I connecting to brane II, we can have:

$$
\Omega_{I, I I}=\exp \left(i \pi \frac{\tau^{i} x_{i}}{2 r}\right) \quad r \rightarrow \infty .
$$

This can happen in the presence of a single soliton solution as we now describe.

In $4+1$ dimensions, consider the gauge-invariant 5-vector

$$
Q^{A}=\frac{g^{2}}{32 \pi^{2}} \epsilon^{A B C D E} F_{B C}^{a} F_{D E}^{a}
$$

It can be seen to satisfy

$$
\partial_{A} Q^{A}=0
$$

leading to the conserved charge

$$
Q=\frac{g^{2}}{32 \pi^{2}} \int d^{4} x \epsilon^{0 \mu \nu \lambda \tau} F_{\mu \nu}^{a} F_{\lambda \tau a}
$$

which must be associated with the soliton solution we are seeking. 
We first consider the limit of very large separation to the branes, $\delta_{I}=-\infty$ and $\delta_{I I} \rightarrow \infty$, where the boundary conditions become irrelevant. In that case, we can write the isolated soliton solution immediately, using 't Hooft's original form for the instanton, by interpreting the Euclidean $x_{4}$ in his formula as $x_{5}$, thus producing a static finite energy monopole solution of the form:

$$
\begin{aligned}
A_{i}^{a} & =\frac{2}{g}\left[\frac{\epsilon_{a i j}[x-r]^{j}+\delta_{a i}\left[x^{5}-r^{5}\right]}{[x-r]^{2}+\rho^{2}}\right] ; \quad(a, i, j)=1,2,3 . \\
A_{5}^{a} & =-\frac{2}{g} \frac{[x-r]^{a}}{[x-r]^{2}+\rho^{2}} \\
A_{0}^{a} & =0
\end{aligned}
$$

where $[x-r]^{2}$ (square brackets) should be understood as

$$
[x-r]^{2}=\left(\eta^{A} \cdot(x-r)_{A}\right)^{2}-(x-r)^{A}(x-r)_{A},
$$

and $\eta^{A}$ is the monopole 5-velocity. The solution is static, centered at $r$ (the square bracket $[x-r]$ are synchronous, i.e., $x_{0}=r_{0}$ ), and we have fixed a particular gauge orientation of this solution, where the hedgehog component is chosen to be $A_{5}^{a}$.

This solution is "self-dual" in the sense that:

$$
\eta^{A} \widetilde{F}_{A B C}^{a}=F_{B C}^{a},
$$

so that the integer-valued topological index is the spatial integral of the charge density of the current $Q_{A}$ in the monopole rest-frame.

This solution can be written in the more compact form, following [9]:

$$
A_{A}^{a} \frac{\tau^{a}}{2}=\frac{2}{g} \bar{\sigma}_{A B} \Pi^{-1} \partial_{B} \Pi \quad \Pi=\left([x-r]^{2}+\rho^{2}\right)
$$

where the $\bar{\sigma}_{A B}$ are antisymmetric and are related to the 't Hooft matrices as:

$$
\begin{aligned}
\bar{\sigma}_{i j}=\epsilon_{a i j} \frac{\tau^{a}}{2 i} & a, i, j=1,2,3, \\
\bar{\sigma}_{5 i}=-\frac{\tau^{i}}{2 i} ; & \bar{\sigma}_{0 A}=0 .
\end{aligned}
$$

In the form for $\Pi$ of eq.(2.11) the monopole charge, which is the 4-volume integral over $Q_{0}$, the "Pontryagin index," can be written as the surface integral 
at infinity over a gauge-dependent current. One can write a gauge equivalent solution! multiplying $\Pi$ by $[x-r]^{-2}$, which does not change the action or the global Pontryagin index, hence:

$$
A_{A}^{a} \frac{\tau^{a}}{2}=\frac{2}{g} \bar{\sigma}_{A B} \Pi^{-1} \partial_{B} \Pi \quad \Pi=\left(1+\frac{\rho^{2}}{[x-r]^{2}}\right)
$$

This latter solution should be viewed as "punctured," in that the singularities are not integrated; the Pontryagin index is given by the small surface integral surrounding the singularity, and the surface at infinity gives no contribution to the charge. This form of the solution is, in a sense, a localizeable monopole, localized at $r$ of size $\rho$. This latter form is most useful for generalization to solutions with the proper brane boundary conditions.

The energy for this static solution is given by the usual expression for the action of a $3+1$ instanton:

$$
S=-\frac{1}{4} \int d x^{4} F_{A B}^{a} F_{A B}^{a}=-\frac{8 \pi^{2} M_{c}}{g_{0}^{2}}
$$

where $F_{A B}^{a}$ are the field strengths. While this expression was the Euclidean action for the instanton, it now plays the role of the mass of our static solution. Hence, this describes a static, topologically stable, magnetic monopole solution with a mass of $8 \pi^{2} M_{c} / g_{0}^{2}$. and thus represents the nontrivial $\Pi_{3}(S U(2))$. This is the relevant homotopy for a monopole in $4+1$ dimensions, the analogue of $\Pi_{2}(G / U(1))$ in $3+1$ dimensions.

The monopole solutions of eq.(2.8, 2.11, 2.13) are only consistent with the boundary conditions in the large $\delta$ limit. Fortunately, one can readily generalize these to multi-monopole solutions along an infinite $x^{5}$ line $[7,8]$. This allows us to use the method of images to implement the boundary conditions.

Consider, first for simplicity, brane I to be at the origin, $x^{5}=0$, hence $\delta_{I}=0$ and brane II at infinity, $\delta_{I I}=\delta \rightarrow \infty$. We locate a monopole at $r=$ $\left(r^{0}, 0,0,0, r^{5}\right)$. We must have that $A_{5}\left(x^{5}\right)=-A_{5}\left(-x^{5}\right)$ and $A_{i}\left(x^{5}\right)=A_{i}\left(-x^{5}\right)$ to satisfy the gauge field boundary conditions described above. Thus, we need to place the appropriate image monopole at $r^{\prime}=\left(r^{0}, 0,0,0,-r^{5}\right)$, with identical

\footnotetext{
${ }^{1}$ This involves a conformal inversion, as well, and is not gauge equivalent if conformal breaking effects, e.g., quantum loops, are considered.
} 


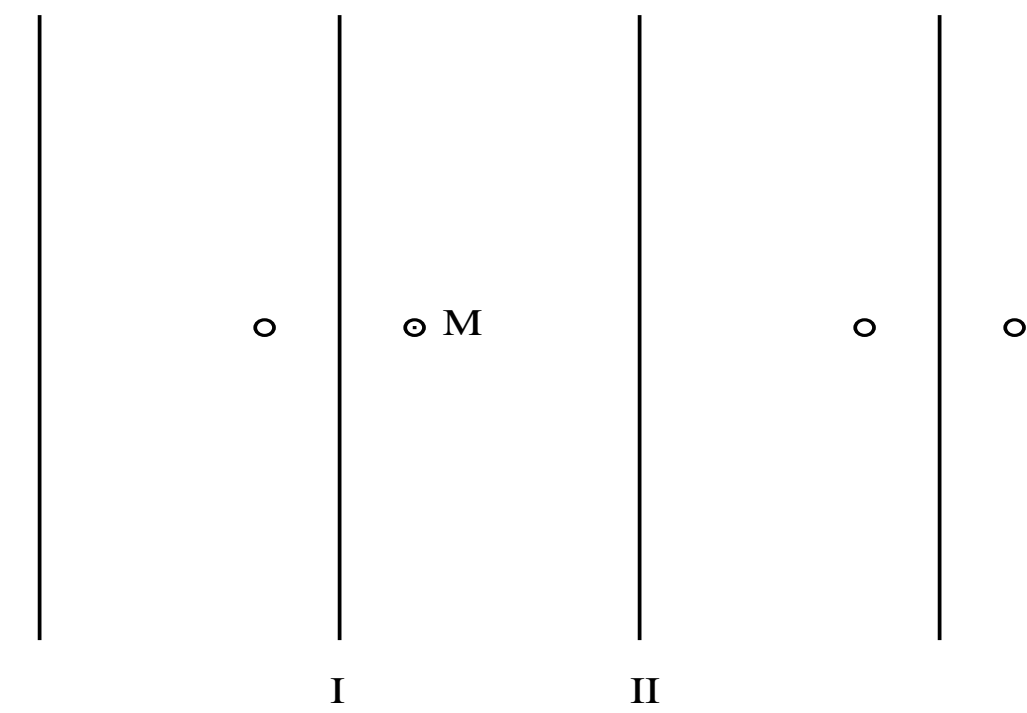

Figure 1: The monopole and its nearest neighbor image domains. The solution is self-dual, following from the Witten solution for an infinite number of instantons on a line. Self-duality implies there is no dependence upon $r^{5}$ of the monopole energy.

gauge orientation. These conditions are satisfied if we require $\partial_{5} \Pi=0$ when $x^{5}=0$. We thus choose:

$$
A_{A}^{a} \frac{\tau^{a}}{2}=\frac{2}{g} \bar{\sigma}_{A B} \Pi^{-1} \partial_{B} \Pi \quad \Pi=\left(1+\frac{\rho^{2}}{[(x-r)]^{2}}+\frac{\rho^{2}}{\left[\left(x-r^{\prime}\right)\right]^{2}}\right)
$$

This self-dual solution represents two identical monopoles, valid everywhere, through the physical region $x^{5}>0$.

We now bring in the second brane from infinity, so that $\delta_{I I}=\delta<\infty$. With the source monopole at $r=\left(r^{0}, 0,0,0, r^{5}\right)$, we now require an infinite set of image monopoles at $r_{n}^{\prime}=\left(r^{0}, 0,0,0,-r^{5}+2 n \delta\right)$, and $r_{n}=\left(r^{0}, 0,0,0, r^{5}+2 n \delta\right)$, all with identical gauge orientations (see Fig.(1)). Hence:

$$
A_{A}^{a} \frac{\tau^{a}}{2}=\frac{2}{g} \bar{\sigma}_{A B} \Pi^{-1} \partial_{B} \Pi ; \quad \Pi=\left(1+\sum_{n=-\infty}^{\infty} \frac{\rho^{2}}{\left[x-r_{n}\right]^{2}}+\sum_{n=-\infty}^{\infty} \frac{\rho^{2}}{\left[x-r_{n}^{\prime}\right]^{2}}\right)
$$

The solution is self-dual in the sense of eq.(2.10). This insures that the energy 
is independent of $r_{5}$, i.e., at this level there is no nontrivial interaction potential with the brane (we will see that this is modified by instantons below). The boundary conditions by themselves imply that a monopole bounces off the brane with unit S-matrix. A brane-monopole collision can be viewed as the exchange of the monopole with its image. For such a bounce off the brane the net change in $\int_{I} d^{4} x F \widetilde{F}=0$ since the orientation of the surface integral over the right monopole upon contact with the brane is opposite to that on the left.

Since the $4+1$ quantum theory is nonrenormalizable, it must be viewed as a cut-off field theory, implying strong cut-off dependence of the physical quantities (one of the nagging questions posed in the introduction). Normally, one views the cut-off in extra-dimensional models as a finite limit on the KK-mode sum. How does this regularization affect the monopole solution we have described? The solution involves Fourier components of arbitrarily large momentum, and one must in principle, discretize these with an upper bound $<M_{s}$, where $M_{s}$ is the "string scale," and typically $M_{c}<<M_{s}$.

The presence of $M_{s}$ obstructs the inversion transformation used to arrive at the periodic solution. The solution cannot be valid on distance scales $\mid x-$ $r \mid<1 / M_{s}$. The large-distance topological behavior remains intact, so solutions such as eq.(2.13), which enforce the topological charge as the surface integral at infinity, remain valid.

We would expect that the string-cut-off places a lower bound on, and discretizes, the physical sizes of our solutions with $\rho>1 / M_{s}$. Moreover, the scale breaking implies that the monopole will have a prefered mass that is dependent upon the size modulus. If the high energy theory has a strong coupling behavior, we would expect stable monopoles with $\rho \sim 1 / M_{s}$, and $M \sim M_{c}$, since $8 \pi^{2} / g_{0}^{2}\left(M_{s}\right) \leq 1$. We also expect stable monopoles with $\rho \sim 1 / \Lambda_{Q C D}$, and $M \sim M_{c}$, since $8 \pi^{2} / g_{0}^{2}\left(\Lambda_{Q C D}\right) \leq 1$.

Let us now consider what happens when we incorporate fermions as modes confined to exist only on brane I. On the brane the axial current satisfies the anomaly relation:

$$
\partial_{\mu} j^{\mu A}=\frac{N g^{2}}{32 \pi^{2}} F_{\mu \nu}^{a} \widetilde{F}^{a \mu \nu}
$$


For a Euclidean 4-volume we have Stokes theorem:

$$
\int_{V} d^{4} x \partial_{\mu} j^{\mu A}=\int_{\partial V} d \mathcal{A}_{3}^{\mu} j_{\mu}^{A}
$$

where $\mathcal{A}_{3}$ is the 3 -surface bounding the Euclidean 4 -volume on the brane. Now, the anomaly is the fifth component of the Q-current flowing off the brane:

$$
\frac{N g^{2}}{32 \pi^{2}} F_{\mu \nu}^{a} \widetilde{F}^{a \mu \nu}=N \chi^{A} Q_{A}
$$

hence:

$$
\int_{\partial V} d \mathcal{A}_{3}^{\mu} j_{\mu}^{A}=N \int_{V} d^{4} x \chi^{A} Q_{A}=N \int_{V} d^{5} x \partial^{A} Q_{A}-N \int_{\partial V}^{\prime} d \mathcal{A}_{4}^{A} Q_{A}
$$

the latter integral on the rhs is over the 4-area bounding a 5-volume, excluding the patch on the brane. The minus sign is due to the sense of the area pointing out of the 5-volume. Using the conservation of $Q$ we thus have:

$$
\int_{\partial V} d \mathcal{A}_{3}^{\mu} j_{\mu}^{5}=-N \int_{\partial V}^{\prime} d \mathcal{A}_{4}^{A} Q_{A}
$$

This says that axial current can flow into a region of the brane and disappear. It becomes a charge associated with the monopole off the brane. This is mediated by the presence of a nonzero expectation value of $F \tilde{F}$ on the brane. This leads to the interpretation of instantons as emission and absorption vertices for monopoles. The effective Lagrangian for the monopole, treated as a quantum particle, together with the brane-instanton interactions will give a modified picture of the $U(1)$ and $\theta$ problems.

\section{Instantons, $\theta$-term, and $U(1)$ Problem}

In the previous section, we have demonstrated the existence of monopole-like solutions of the Yang-Mills field equations in the bulk. Our picture also gives a reinterpretation of the instantons of the $3+1$ theory.

The traditional instanton remains as a Euclidean solution with the supplemental condition that $A_{5}^{a}=0$, and the solution is independent of $x^{5}$. Hence, it immediately satisfies the brane boundary conditions. If we pass to Euclidean time, $x^{0} \rightarrow i \tilde{x}^{0}$, the solution can be viewed as a one-dimensional Euclidean 


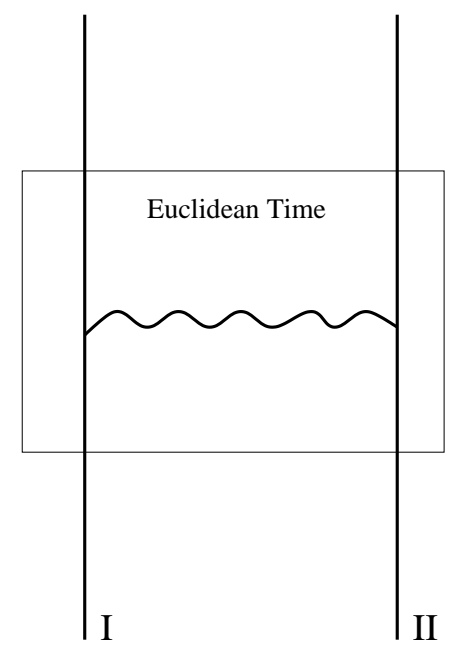

Figure 2: The traditional instanton is a cylindrical solution, analogous to a vortex, independent of $x^{5}$, in $4+1$ Euclideanized space-time.

"vortex," which emanates from brane I and terminates on brane II. The instanton removes one unit of the associated charge from brane I and deposits it on brane II as shown in Fig.(2). Deformed solutions, as in Fig.(3) must exist also, and must be continuously connected energetically to the straight line vortex limit. The mixing of $x^{5}$ with $\tilde{x}^{0}$ under deformation implies that the instanton can be viewed as a vertex for emission of the monopole from the brane as in Fig.(3). The monopole in the Euclideanized time situation is identical to that in the Minkowski situation, and the monopole rotated in Euclidean space-time becomes the instanton.

If we cut through an instanton vortex exchanged between the branes as in Fig.(4) we see that we have cut through the exchange propagator of a monopole. Hence, the traditional instanton in $3+1$ now appears as a "t-channel" exchange of the monopole between the two branes in $4+1$. This picture gives us a dual interpretation of instanton physics: Whereas the amplitude for the instanton in Euclideanized $3+1$ takes the form $\sim \exp \left(-8 \pi^{2} / g_{0}^{2}\right)$, the t-channel monopole exchange in $4+1$ involves a Yukawa factor $\sim \exp (-M \delta)=\exp \left(-8 \pi^{2} / g_{0}^{2}\right)$. This reinterpretation can provide, as we see below, an equivalent resolution of $U(1)_{A}$ 


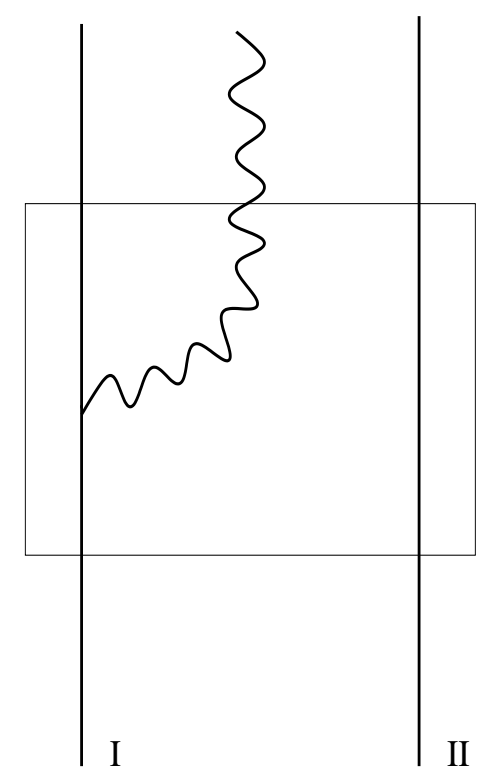

Figure 3: Instanton as emission vertex for a monopole off the brane into the bulk.

problem. An overall CP-violating $\theta$ angle still remains in the present case.

The emission vertex must be consistent with the description of a leakage of current off brane I into the bulk. The $\theta$-term in $4+1$ is no longer Lorentz invariant, and can be viewed as the current source from the brane into the bulk. In the presence of this source, the monopole produces a coherent classical field between the branes. This field, produced on one, brane is exponentially damped as $\sim \exp (-M \delta)$ as it "shines" on the other brane, which reproduces the traditional instanton tunneling probability, $\sim \exp \left(-8 \pi^{2} / g_{0}^{2}\right)$. While the monopole field allows us to remove, by phase redefinition, any $\theta_{i}$ term on brane $\mathrm{i}$, there remains a net $\theta_{I I}-\theta_{I} \equiv \theta$ term overall in the physics, and the overall $\theta$ angle remains. The monopole t-channel exchange reproduces the effect of the traditional instanton. The ultimate removal of the residual $\theta$ term would require, e.g., an axion (which may arise on brane II in isolation from fermionic structure on brane I).

A quantitative description of this picture involves setting up an effective Lagrangian describing the brane-monopole dynamics. To that effect, we represent the monopole by a complex scalar field $F$ in the bulk. Although it should 


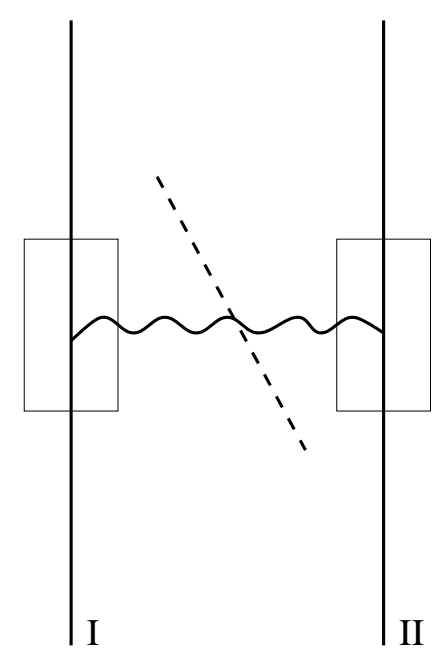

Figure 4: Cutting through instanton reveals monopole exchange. The emission vertices can be shrunk to localized (localized in $x^{5}$, of extent $\epsilon$ ) Euclidean patches on the brane, the $\exp \left(-8 \pi^{2} M_{c} \epsilon / g_{0}^{2}\right)$ tunneling amplitude for a patch approaching unity. The full $\exp \left(-8 \pi^{2} / g_{0}^{2}\right)$ tunneling amplitude factor is then restored by the Yukawa exchange amplitude of the monopoles, $\propto \exp (-M \delta)$.

be parameterized by various moduli, including the size modulus $\rho$, we suppress these collective parameters at present (we are considering monopoles of size $\sim 1 / \Lambda_{Q C D}$. We start with an effective $4+1$ free Lagrangian density in the bulk:

$$
\mathcal{L}_{F}=\partial_{A} F^{\dagger} \partial^{A} F-M^{2} F^{\dagger} F
$$

The $x^{5}$-periodic gauge-field monopole solution implies supplemental periodic boundary conditions on $F: F$ must be symmetric under $x^{5} \rightarrow 2 \delta_{m}-x^{5}$, for a brane located at $x^{5}=\delta_{m}$. Note that $F$ does not satisfy Neuman conditions, since the field essentially parametrizes the centers of gauge solitons. The current:

$$
J^{A}=\frac{i}{2} F^{\dagger} \stackrel{\leftrightarrow}{\partial}^{A} F \sim Q^{A}
$$

must be identified with the topological current, $Q^{A}$. This current is conserved in the bulk, but will have sources at the branes since our effective Lagrangian must represent eq.(2.21), i.e., the change in the $Q_{5}$ current component upon 
traversing the brane must be given by the current source term:

$$
\left.\frac{i}{2} F^{\dagger} \stackrel{\leftrightarrow}{\partial}{ }^{5} F\right|_{\text {brane+ }}-\left.\frac{i}{2} F^{\dagger} \stackrel{\leftrightarrow}{\partial}{ }^{5} F\right|_{\text {brane- }}=<\frac{N g^{2}}{32 \pi^{2}} F_{\mu \nu}^{a} \widetilde{F}^{a \mu \nu}>
$$

Note that, in QCD:

$$
<\frac{g^{2}}{32 \pi^{2}} F_{\mu \nu}^{a} \widetilde{F}^{a \mu \nu}>=\frac{\delta}{\delta \theta} V(\theta) \approx-\tilde{\kappa} e^{-8 \pi^{2} / g_{0}^{2}} \Lambda_{Q C D}^{4} \sin \theta
$$

where $V(\theta)$ is the the $\theta$-potential (this is essentially $i \hbar \ln$ (Feynman path integral) in the presence of instantons). Here $\tilde{\kappa}$ is the effective coupling constant involving the full instanton configuration sum, the determinant over small oscillations, etc. In order to describe these effects, we must supplement the free effective action for $F$ with a source term that describes the monopole emission from the brane into the bulk.

In the $4+1$ theory there is no Lorentz invariant $\theta$-term. The $\theta$-term physically depends upon the definition of the branes, e.g., their fermionic content, etc. For example, if fermions are localized on brane I, we must allow for a localized $\theta$ term on the brane, which appears as a "topological current" source:

$$
\mathcal{L}_{\theta}=\frac{\theta_{1} N g_{0}^{2}}{32 \pi^{2}} \int d^{5} x \delta\left(x^{5}\right) \chi^{A} \epsilon_{A B C D E} F^{a B C} F^{a D E}
$$

where $\chi^{A}$ is the spacelike 5-vector normal to the brane pointing into the bulk. Alternatively, we can view the brane as a classical object with an orientation defined by $\chi_{A}$, and this is simply an allowed effective term 1 .

In order to reproduce the essential current algebra embodied in eq.(3.24), we need to include the amplitude for emission of monopoles from the branes. This term should be proportional to $e^{i \theta_{i}}$. Shifting in QCD $\theta_{i} \rightarrow \theta_{i}+\lambda$ generates the divergence of the axial current; the corresponding shift in $F \rightarrow \exp (-i \lambda) F$

\footnotetext{
${ }^{2}$ We'll conventionally put the full Standard Model fermion structure on brane I. Alternatively, with fermions in the bulk, which we do not consider presently, we must allow for a more general bulk-filling $\theta$ term:

$$
\mathcal{L}_{B u l k}=\theta \int d^{5} x M_{c} \chi^{A} \epsilon_{A B C D E} F^{a B C} F^{a D E}
$$

This latter term will imitate a $\theta$-term in the effective low energy $3+1$ theory, when appropriate boundary conditions are implemented. We will restrict our attention to the case of $\mathcal{L}_{\theta}$.
} 
generates the divergence of the $Q^{A}$ Noether current. This leads us to postulate the amplitude for emission of monopoles from the brane:

$$
\mathcal{L}_{\text {emission }}=\sum_{m=I, I I} \delta\left(x^{5}-\delta_{m}\right) \Lambda_{Q C D}^{2} M^{1 / 2} \tilde{g}_{m} e^{i \theta_{m}} F+h . c .
$$

where $\tilde{g}$ is a dimensionless parameter, and $\theta_{m}$ the $m$ th brane's $\theta$ angle. The dimensionality of the coefficient is chosen to match the dimensionality of the $F$ field $(3 / 2)$ and to satisfy consistency with the instanton result. The $\sqrt{M}$ factor is a normalization factor in $F$ consistent with the proper normalization of a single quantum of $F$ carrying a unit topological charge.

How would we compute $\tilde{g}_{m}$ from first principles? We give only a heuristic argument presently. The emission vertex is an instanton patch on the brane of finite length $\epsilon$ in space, $x^{5}$, i.e., the instanton, which is emitted from brane I, terminates on the monopole at $x^{5}=\epsilon$. The nonperturbative factor in the action for this is $\sim \exp \left(\int_{0}^{\epsilon} d x^{5} 8 \pi^{2} / g^{2}\right) \sim \exp \left(8 \epsilon M_{c} \pi^{2} / g_{0}^{2}\right)$. This approaches unity for $\epsilon \rightarrow 0$. Since we are exchanging a large monopole, the relevant gauge coupling constant feels large transverse distances in the Euclidean $3+1$ dimensions, and is presumably not suppressed by asymptotic freedom as $\epsilon \rightarrow 0$. The usual instanton amplitude prefactor, however, consisting of the bosonic determinant for small oscillations around the instanton solution, and a 't Hooft determinant over spin $-1 / 2$ fields, would still be present. Hence, $\tilde{g}_{m}$ is computed as if we were calculating the action for an instanton, but we discard the nonperturbative factor. The factor is local to the $m$ th brane, as it must be because the fermionic content of a given brane is arbitrary. The nonperturbative factor, as we stated above, returns through the $t$-channel exchange of the monopole. For the emission of small monopoles, or on-shell monopoles, we require a smaller transverse instanton, and the relevant coupling constant $g_{0}$ becomes $g_{0}(\rho)$.

Neglecting fermions temporarily, we consider the effective action

$$
\mathcal{L}_{T}=\mathcal{L}_{F}+\mathcal{L}_{\text {emission }}
$$

from which we derive the equation of motion for the monopole field:

$$
0=\partial^{A} \partial_{A} F+M^{2} F-\sum_{m=I, I I} \tilde{g}_{m} e^{-i \theta_{m}} \Lambda_{Q C D}^{2} M^{1 / 2} \delta\left(x^{5}-\delta_{m}\right)
$$


We seek a solution is of the form:

$$
\begin{aligned}
& F=\alpha \exp \left(-M x^{5}\right)+\beta \exp \left(-M\left(\delta-x^{5}\right)\right) \quad 0<x^{5} \leq \delta ; \\
& F=\alpha \exp \left(M x^{5}\right)+\beta \exp \left(-M\left(\delta+x^{5}\right)\right) \quad-\delta<x^{5} \leq 0 ; \\
& F=\alpha \exp \left(-M\left(2 \delta-x^{5}\right)\right)+\beta \exp \left(M\left(\delta-x^{5}\right)\right) \quad \delta \leq x^{5}<2 \delta,
\end{aligned}
$$

where brane I has been set at $x^{5}=0$ and brane II at $x^{5}=\delta .5$

This solution implements the even periodicity of the underlying monopole gauge field solution, which implies reflection symmetries about the branes. The solution is infinitely periodic as dictated by the infinite system of images, however, we require it only in these three domains to obtain $\alpha$ and $\beta$. $\alpha$ and $\beta$ are determined by integrating through the source delta-functions:

$$
\begin{aligned}
& \left.\frac{\partial F}{\partial x^{5}}\right|_{x^{5}=0^{+}}-\left.\frac{\partial F}{\partial x^{5}}\right|_{x^{5}=0^{-}}=-\tilde{g}_{1} \Lambda_{Q C D}^{2} M^{1 / 2} e^{-i \theta_{1}} \\
& \left.\frac{\partial F}{\partial x^{5}}\right|_{x^{5}=\delta^{+}}-\left.\frac{\partial F}{\partial x^{5}}\right|_{x^{5}=\delta^{-}}=-\tilde{g}_{2} \Lambda_{Q C D}^{2} M^{1 / 2} e^{-i \theta_{2}}
\end{aligned}
$$

Hence, we have:

$$
\begin{aligned}
\alpha & =\frac{\Lambda_{Q C D}^{2} e^{-i \theta_{1}}}{4 M^{1 / 2} \sinh (M \delta)}\left(\tilde{g}_{1} e^{M \delta}+\tilde{g}_{2} e^{i \theta}\right) ; \\
\beta & =\frac{\Lambda_{Q C D}^{2} e^{-i \theta_{1}}}{4 M^{1 / 2} \sinh (M \delta)}\left(\tilde{g}_{1}+\tilde{g}_{2} e^{i \theta+M \delta}\right) ;
\end{aligned}
$$

where:

$$
\theta \equiv \theta_{1}-\theta_{2}
$$

The full solution is therefore:

$$
F=\frac{\Lambda_{Q C D}^{2} e^{-i \theta_{1}}}{4 M^{1 / 2} \sinh (M \delta)}\left[\left(\tilde{g}_{1} e^{M \delta}+\tilde{g}_{2} e^{i \theta}\right) e^{-M x^{5}}+\left(\tilde{g}_{1} e^{-M \delta}+\tilde{g}_{2} e^{i \theta}\right) e^{M x^{5}}[3] .35\right)
$$

Note that the overall factor of $e^{-i \theta_{1}}$ is irrelevant, and can be absorbed into a redefinition of $F$, and only the net $\theta=\theta_{1}-\theta_{2}$ remains in the physics. This

\footnotetext{
${ }^{3}$ We remark that we could allow an overall phase factor, $\exp \left(i \omega / f_{\omega}\right) F$, where $\omega$ depends upon $x^{\mu}$ and acts like a (pseudo) Nambu-Goldstone boson; however, the boundary conditions, eq. 3.32 , force $\omega=0$, and small oscillations in $\omega$ are not relevant to the physics in this case (see discussion below eq.(3.42)).
} 
solution yields the nontrivial profile of the monopole effective field $F$ between two branes with arbitrary $\theta$ terms. It may be viewed as the manifestation of the vacuum condensate of monopoles between the branes.

We can readily verify the boundary condition on the current, e.g., at brane $\mathrm{I}, x^{5}=0$ :

$$
\left.\frac{i}{2} F^{\dagger} \stackrel{\leftrightarrow}{\partial}{ }^{5} F\right|_{0+}-\left.\frac{i}{2} F^{\dagger} \stackrel{\leftrightarrow}{\partial}{ }^{5} F\right|_{0-}=-\frac{\tilde{g}_{1} \tilde{g}_{2} \Lambda_{Q C D}^{4}}{2 \sinh (M \delta)} \sin \theta \sim-\left.\tilde{g}_{1} \tilde{g}_{2} e^{-8 \pi^{2} / g_{0}^{2}} \Lambda_{Q C D}^{4} \sin \theta\right|_{M \delta>>1}
$$

This illustrates consistency of the two pictures, with $\tilde{\kappa}=\tilde{g}_{1} \tilde{g}_{2}$. Note the factorization of the overall effect into the contribution from the two emission vertices of the two branes. The current discontinuity is driven by the overall $\theta$ angle, as the QCD instanton picture predicts.

We now examine the low energy effective Lagrangian including contributions from both branes and the bulk. We substitute our solution for $F$ back into the action and integrate over $x^{5}$. Since our solution is infinitely periodic, the brane contributions to the action must be viewed as shared between the physical domain $0 \leq x^{5} \leq \delta$ and the nearest neighbor image domains. Hence, each brane contribution to the action in the physical domain $0 \leq x^{5} \leq \delta$ receives a factor of $1 / 2$, while the bulk contribution comes from integrating eq.(3.22) over $0 \leq x^{5} \leq \delta$. These contributions have the same structure and the overall result is:

$$
\mathcal{L}_{T}=\frac{1}{4}\left(F_{\mu \nu}^{a}\right)^{2}+\frac{\Lambda_{Q C D}^{4}}{4 \sinh (M \delta)}\left[\left(\tilde{g}_{1}^{2}+\tilde{g}_{2}^{2}\right) \cosh (M \delta)+2 \tilde{g}_{1} \tilde{g}_{2} \cos (\theta)\right]
$$

This produces a nontrivial potential in $\theta$, the residual net $\mathrm{CP}$-angle. In the large $M \delta$ limit we obtain:

$$
L \approx \frac{1}{4}\left(F_{\mu \nu}^{a}\right)^{2}+\Lambda_{Q C D}^{4}\left[\frac{1}{4}\left(\tilde{g}_{1}^{2}+\tilde{g}_{2}\right)+\tilde{g}_{1} \tilde{g}_{2} e^{-M \delta} \cos \theta+O\left(e^{-2 M \delta}\right)\right]
$$

Note that we have interaction self-energy terms $\propto\left(\tilde{g}_{1}^{2}+\tilde{g}_{2}\right)$ which have no $\theta$ dependence. Moreover, we recover the $\theta$-potential in QCD $\propto \Lambda_{Q C D}^{4} \tilde{g}_{1} \tilde{g}_{2} \exp (-M \delta) \cos \theta$, where the factor $\exp (-M \delta)=\exp \left(-8 \pi^{2} / g_{0}^{2}\right)$ now reflects the duality between the 't Hooft tunneling amplitude and the t-channel exchange amplitude. This result implies eq.(3.36) identically upon differentiating wrt $\theta$. 
Including fermions, the emission vertex from brane I must include the 't Hooft determinant as:

$$
\delta\left(x^{5}\right) \tilde{g}_{1}(\Lambda)^{p} \sqrt{M} \operatorname{det} \bar{\psi}_{L} \psi_{R} e^{i \theta_{1}} F+h . c .
$$

If we include 3 flavors of light quarks, then $p=-7$. Normal QCD chiral dynamics will condense fermions, making a chiral Lagranagian of pseudoscalar mesons. We are interested in the fate of the $\eta^{\prime}$. hence, we can "bosonize" the "t Hooft determinant in the usual way, replacing it with the $\eta^{\prime}$ field:

$$
\operatorname{det} \bar{\psi}_{L} \psi_{R} \rightarrow c \Lambda^{9} \exp \left[\frac{3 i \eta^{\prime}}{\sqrt{6} f_{\pi}}\right]
$$

The $\eta^{\prime}$ mass comes only from the brane contribution. If we redefine the monopole $F$ field as $F \rightarrow \exp \left(i \theta_{1}+3 i \eta^{\prime} / \sqrt{6} f_{\pi}\right) F$ (this has insignificant effect upon the $\eta^{\prime}$ kinetic term), then we see that the preceding analysis goes through, yielding a potential which involves the $\eta^{\prime}$ :

$$
L \approx \frac{1}{4}\left(F_{\mu \nu}^{a}\right)^{2}+c \tilde{g}_{1} \tilde{g}_{2} \Lambda_{Q C D}^{4} \exp \left(-8 \pi^{2} / g_{0}^{2}\right) \cos \left(\sqrt{3 / 2} \eta^{\prime} / f_{\pi}+\theta\right)
$$

This provides the usual resolution to the $U(1)$ problem, elevating the mass of the $\eta^{\prime}$. It also shows that the usual $\theta$ problem remains, since other terms in the Lagrangian will contain $\eta^{\prime}$ without the $\theta$ angle, and we cannot simply remove the CP-violation by a shift in the $\eta^{\prime} \mathrm{VEV}$, e.g., the interference terms will lead to a nonzero neutron electric dipole moment.

The solution we have obtained is the technically appropriate one for the specific two brane model under consideration. The physics can be better appreciated in various limits. What happens, for example, if we attempt to remove brane II from the discussion? Of course, brane II is serving the role of decoupling the high energy KK modes, and providing a nonzero monopole mass. That is, we cannot simply take $\delta \rightarrow \infty$ or we would have a massless monopole, and a $4+1$ bulk theory. We can consider, however, the limit $\tilde{g}_{2} \rightarrow 0$. Then the $\theta$ angle disappears, and also the solution to the $U(1)$ problem. What has happened? In this case the physics is controlled by brane I. The effective Lagrangian is then $\propto \Lambda^{2} \sqrt{M} e^{i \theta_{1}} F+h . c$. . On brane I, $F$ acquires the VEV, $\sim\left(\Lambda^{2} / \sqrt{M}\right) e^{i \omega / f_{\omega}}$, where $\omega$ is a free phase factor (a Nambu-Goldstone boson) that depends upon $x^{\mu}$ 
and $f_{\omega} \sim \Lambda^{2} / M$. Thus the effective potential in $\omega$ is $\sim \Lambda^{4} \cos \left(\theta_{1}+\omega / f_{\omega}\right)$. The $\theta_{1}$ angle is now dynamically relaxed to zero by the $\omega$ field. This simply restates the obvious fact that the $\theta_{1}$ angle is removable by a phase redefinition of $F$. In this case $\theta_{1}$ represents the applied or "thermal cathode current" of topological charge into the bulk. The monopole establishes a compensating field configuration such that the total current flow, $\propto \sin \left(\theta_{1}+\omega / f_{\omega}\right)$ relaxes to zero. This is analogous to a "space-charge" limited cathode current; the current is quenched by the accumulation of monopoles in the bulk. If we include the $\eta^{\prime}$ in this case, we obtain the potential $\sim \Lambda^{4} \cos \left(3 \eta^{\prime} / \sqrt{6} f_{\pi}+\omega / f_{\omega}\right)$. This is analogous to what happens with an axion (there are, of course, additional terms which give the $\eta^{\prime}$ a mass of order $m_{\pi}$ without the $\omega$ field in linear combination). However, in the case of the axion we have $f_{\text {axion }}>>f_{\pi}$; hence a see-saw mechanism is always at work in axion physics which leaves the $\eta^{\prime}$ heavy and makes the axion light. In the present case, $f_{\omega} \sim \Lambda^{2} / M<<f_{\pi}$, and the field $\omega$ undergoes large amplitude excursions in a single quantum state, i.e. $\omega$ is a random phase. Hence, integrating out $\omega$ washes out the contribution to the $\eta^{\prime}$ mass, and the unwanted fourth $U(1)_{A}$ Nambu-Goldstone boson returns.

In our two brane model there is a net $\mathrm{CP}$-violation parameterized by $\theta=$ $\theta_{1}-\theta_{2}$, which is physically analogous to two different thermal cathode currents on the branes. The $\omega$ field cannot relax this linear combination to zero, and an equlibrium monopole condensate is formed which conducts the net current $\sim \tilde{g}_{1} \tilde{g}_{2} e^{-M \delta} \sin \left(\theta+3 \eta^{\prime} / \sqrt{6} f_{\pi}\right)$ through the bulk. The $\eta^{\prime}$ acquires a mass as this current is not quenched by $\omega$.

\section{Discussion and Conclusions}

We have initiated a study of certain gauge field solitons that occur in higher dimensional imbeddings of the Standard Model with gauge fields in the bulk. We have used the method of images and the multi-instanton solution in a line to obtain self-dual solutions consistent with brane boundary conditions. The monopoles have mass $8 \pi^{2} M_{c} / g_{0}^{2}$ where $M_{c}=1 / \delta$ is the inverse compactification scale.

We have considered one application, the reinterpretation of instantons as 
the emission/absorption vertices of monopoles from the brane into the bulk. The $U(1)_{A}$ problem is then resolved by considering the monopole condensate which forms in the bulk between branes. The $\theta$ problem remains, requiring the usual resolution, e.g., in terms of an axion. We remark that, if strong dynamics at high energies lead to a large $\sim M_{\text {string }}$ condensate in $F$ for small instantons $\rho \sim 1 / M_{c}$, then the phase field $\omega$ may act like an axion in certain cases. This would be a purely hadronic axion, i.e., one which couples only to $F_{\mu \nu}^{a} \tilde{F}_{Q C D}^{a \mu \nu}$ but not to $F_{\mu \nu} \tilde{F}_{Q E D}^{a \mu \nu}$. This may ultimately be natural in these schemes, and would alleviate many of the restrictive phenomenological constraints on $f_{\text {axion }}$.

Our reinterpretation of instantons as the exchange of monopoles at low energies suggests an analogy to the AdS-CFT correspondence. The, the boundary theory of $d=0$ pointlike instantons is seen to be equivalent to the compactified bulk theory of $d=1$ worldline monopoles at low energies.

Phenomenologically, at sufficiently high energies, monopoles would be produced in collisions on the brane. For example, $g+g \rightarrow F$ is possible if the $g g$ configuration can produce a nonzero $F_{\mu \nu} \tilde{F}^{\mu \nu}$. Nontopological pair production $g+g \rightarrow F^{\dagger}+F$ can also occur. We expect that the rates may be enhanced at sufficiently high energy because the modulus $\rho$ must be summed, and a very large number of states are available (much like the KK mode production at very high energies of gravitons where the many KK modes compensate the suppressed coupling).

In the case that $S U(2)$ is identified with $S U(2)_{L}$, the monopole charge corresponds to $B+L$, by comparison with the fermionic current anomalies. In this case a similar picture of instanton mediated baryon number violation would apparently arise, involving corresponding $B+L$ carrying monopoles in the bulk, and a monopole condensate. Interestingly, the $B+L$ violation rates are proportional to $\exp (-M \delta)$, and could be enhanced if the branes are brought closer together. Could this have implications for a theory of cosmological baryogenesis?

There are a large number of issues to address beyond what has been considered here. Spontaneous symmetry breaking can a lead to a new class of monopoles involving the (effective) Higgs field. In the BPS limit, the construction employed here might extend to writing down self-dual monopoles consis- 
tent with the brane boundary conditions by the method of images. Moreover, the topologies of other objects generalize similarly, e.g., the 't Hooft-Polyakov monopoles become domain walls in one extra dimension; vortices become 3branes; etc. New objects of different homotopy classes are expected as well [10].

Moreover, we might ask what additional dynamics may be involved in extradimensional theories? For example, it is presumably straightforward to extend this effective theory to the Randall-Sundrum model [2], and we might expect an intriguing mixing of scales in that case. Clearly, whatever brane cosmology emerges, the fate of novel monopoles will be an issue, requiring some solution such as inflation. We expect this to be a rich arena for follow-on studies. 


\section{Acknowledgements}

We wish to thank W. Bardeen, E. Weinberg, and M. Moshe for useful comments. One of us (PR) wishes to acknowledge the kind hospitality of the Fermilab theory group where this work originated, and to thank the Aspen Center for Physics for its kind hospitality during the completion of this work.

\section{Bibiliography}

1. I. Antoniadis, Phys.Lett. B246 (1990) 377;

I. Antoniadis, C. Munoz, M. Quiros Nucl. Phys. B397 (1993) 515;

I. Antoniadis, K. Benakli, M. Quiros Phys. Lett. B331 (1994) 313;

J. Lykken, Phys. Rev. D54, 3693 (1996);

I. Antoniadis, S. Dimopoulos and G. Dvali, Nucl. Phys. B516, 70 (1998);

N. Arkani-Hamed, S. Dimopoulos and G. Dvali, Phys. Lett. B429, 263 (1998);

J. Lykken, L. Randall, "The Shape of gravity," hep-th/9908076.

2. L. Randall and R. Sundrum, Phys. Rev. Lett. 83, 3370 (1999);

3. K.R. Dienes, E. Dudas and T. Gherghetta, Phys. Lett. B436, 55 (1998);

K.R. Dienes, E. Dudas and T. Gherghetta, Nucl. Phys. B537, 47 (1999); see also: hep-ph/9807522;

4. M. Dine and N. Seiberg, Phys. Lett. 162B, 299 (1985);

H.-C. Cheng, B. A. Dobrescu and C. T. Hill, "Electroweak symmetry breaking by extra dimensions", hep-ph/0004072 and hep-ph/9912343;

H.-C. Cheng, B. A. Dobrescu and C. T. Hill, Nucl.Phys. B573 597, (2000);

N. Arkani-Hamed, H.-C. Cheng, B. A. Dobrescu and L. J. Hall", "Selfbreaking of the standard model gauge symmetry", hep-ph/0006238';

N. Arkani-Hamed and M. Schmaltz, "Hierarchies without symmetries from extra dimensions," hep-ph/9903417.

5. A.A. Belavin, A.M. Polyakov, A.S. Shvarts, Yu.S. Tyupkin, Phys. Lett. B59 85, (1975) 
6. G. 't Hooft, Phys. Rev. Lett. 37, 8 (1976).

7. E. Witten, Phys. Rev. Lett. 38, 121 (1977).

8. D. J. Gross, R. D. Pisarski, L. G. Yaffe, Rev. Mod. Phys 53, 43 (1981); Many useful results and references are collected herein. Our construction bears a strong resemblance to the finite temperature considerations discussed in this paper.

9. R. Jackiw, C. Nohl and C. Rebbi, Phys. Rev. D15, 1642 (1977).

10. G. Dvali, I. Kogan, M. Shifman, "Topological Effects in our Brane World from Extra Dimensions," NYU-TH-00-06-01, hep-th/0006213. 